\title{
The Effect of Velocity and Interfacial Tension on the Relative Permeability of Gas Condensate Fluids in the Wellbore Region
}

\author{
Henderson G. D., Danesh A., Tehrani D., Peden J. M.
}

Heriot - Watt Univ., U. K.

\begin{abstract}
Copyright 1995, Steering Committee of the European IOR - Symposium.
This paper was presented at the 8th. European IOR - Symposium in Vienne. Austria, May 15 - 17,1995

This papor was selected for presentation by the Steering Committee, following roviow of information contained in an abstract

submitted by the author(s). The paper, as presented hes not been reviewed by the $\$$ teering Committee.
\end{abstract}

\begin{abstract}
High pressure core flood experiments using gas condensate fluids in long sandstone cores have been conducted to determine the effect of flow rate and interfacial tension (IFT) on relative permeability. The experimental data are intended to be applied to near wellbore flow in gas condensate reservoirs, as viscous forces increase over capillary during the course of the tests.
\end{abstract}

A relative permeability rate effect for both gas and condensate phases was observed when using the steady-state and unsteady-state methods. Initial tests were repeated at increasing flow rates which showed that the relative permeability of both phases increased with the increase in flow rate. Increasing the value of IFT between the phases reduced the relative permeability of the gas phase more than the condensate phase, with the relative permeability of both phases continuing to increase at higher flow rates. The influence of core end effects was shown in be negligible at the low IFT conditions used in ti:e tests. The Reynolds number indicated that flow was within the so called laminar regime at all test conditions. The observed rate effect was contrary to that of the conventional non-Darcy flow where the effective permeability decreases with increasing flow rates. Relative permeability tests conducted with conventional gas-oil fluids at similar"test conditions did not exhibit any significant rate effect.

The results highlight the need for appropriate experimental methods and relative permeability relations for gas condensate reservoirs. This study will be particularly applicable to the vicinity of producing wells, where the rate effect on gas relative permeability can significantly affect well productivity. The findings provide previously unreported data on relative permeability and recovery of gas condensate fluids at realistic conditions, which can be used as useful guide-lines for the management of gas condensate reservoirs.

\section{INTRODUCTION}

Until now, when generating laboratory relative permeability data to be applied to now in gas condensate reservoirs, conventional gas-oil unsteady-state/steady-state relative permeability procedures have generally been used $\{1,2,3]$. The assumption being that the flow of condensing fluids could be accurately represented in laboratory core tests using conventional procedures. However; there are fundamental differences between the flow characteristics of condensing and conventional fluids.

As gas condensate reservoirs are produced by either pressure depletion or gas cycling, the reservoir pressure will gradually be reduced to below the dew point, at which stage the liquid saturation will increase from zero by retrograde condensation. Condensate will drop-out in all pore space [4], reducing the flow of gas from the reservoir. This condensate has been shown to be mobile ${ }^{[s]}$, but recovery is generally percieved to be small.

The fluid distributions established in cores during conventional gas-oil displacements are created by injection, resulting in the larger pores containing 
mostly gas while the smaller pores contain mostly oil. During unsteady-state primary drainage tests the initial oil saturation in the core is reduced from $100 \%$ by gas injection. This differs from the process of condensation where the liquid saturation increases from zero. Conventional gas-oil experimental procedures used to generate relative permeability data do not therefore reproduce the fluid distributions created by condensing fluids, nor do they have the associated flow mechanisms $[4,6,7]$.

The most important region of the reservoir where relative permeability data on the flow of gas and condensate is required is in the vicinity of producing wells where flow is governed by surface and shear forces. The reduction in reservoir pressure in this region associated with production can lead to a fall in gas productivity due to an increase in the condensate saturation in the vicinity of the wellbore. The condensate build up arises due to the process of condensation associated with pressure reduction below the dew-point, as well as inflow of condensate from the main reservoir. Reduction in reservoir pressure also increases capillary forces, associated with increasing IFT, which retain the condensate in all pore space. As gas condensate fluids flow towards producing wells, their velocity will also increase as the wellbore is approached due to radial flow, the extent of which is influenced by. the rate of production. The effect of increasing capillary/surface forces (IFT), combined with increasing shear forces (velocity) on gas condensate relative permeability and production in the vicinity of producing wells is far from clear.

The objective of this study was therefore to investigate any sensitivity of gas condensate relative permeability to velocity and IFT. The flow rates used in the tests were chosen to be of particular relevance to the regions of the reservoir in the vicinity of producing wells. Experimental procedures were developed specifically to measure gas condensate relative permeability using long cores, with the experimental procedures ensuring that the distribution of the phases in the core were representative of the fluid distribution in the reservoir.

In order to address the applicability of conventional gas-oil methods to generate relative permeability data for this section of gas condensate reservoirs, conventional displacement tests were conducted at similar experimental conditions and the results were compared to the data generated using condensing fluids. The comparison clearly defines the limitations in the transfer of conventionally generated data to the flow of gas condensate fluids.

The findings highlight the need for the accurate measurement of relative permeability for condensing fluids using the appropriate experimental procedures, whilst also providing guidelines for future reservoir management.

\section{EXPERIMENTAL PROCEDURES}

\section{Core properties}

The core used in all tests was a Berea core, the characteristics of which are shown in Table 1. Tracer analysis of the core prior to the tests had indicated that the core was homogeneous. The core was known to be water-wet, and contained a connate water saturation of $26.4 \%$. The reported gas and condensate/oil saturations for each Test were normalised and reported as a percentage of the hydrocarbon pore volume. The connate water saturation is believed to have remained constant between tests, as the base relative permeability remained unchanged.

\begin{tabular}{|c|c|c|c|c|c|}
\hline Core & $\begin{array}{c}\text { (cm) } \\
\text { Lengh }\end{array}$ & $\begin{array}{c}\frac{(\mathrm{cm})}{\text { Diam }} \\
\text { Diarosity }\end{array}$ & $\begin{array}{c}\frac{\%}{\%} \\
\text { Powi }\end{array}$ & $\begin{array}{c}\text { (md) } \\
\text { Kat Swi }\end{array}$ \\
\hline Berea & 61 & 5.1 & 18.2 & 26.4 & 92 \\
\hline
\end{tabular}

Table 1 Core properties

\section{Rotation of the horizontal core}

The core used in this study was mounted horizontally and continually rotated through 360 degrees for the duration of the tests, in order to minimise the influence of gravitational forces on the fluid distribution. If this procedure was not followed when using condensing fluids, especially at the low IFT Test conditions where gravitational forces are dominant, the lower sections of the core may have filled with condensate before production could occur. This would have resulted in segregated flow of gas and condensate in the core [7].

\section{Test fluid}

Gas condensate Test Fluid

The composition and liquid drop-out of the gas condensate mixture used are shown in Table 2, and Figure 1. The values of interfacial tension (IFT) quoted for the tests were measured in a PVT cell using the gas-liquid interface curvature method [8].

This gas mixture was used in all tests reported, with the low IFT Tests $(0.05 \mathrm{mN} / \mathrm{m})$ being conducted at a pressure of $30.4 \mathrm{MPa}$, and the higher IFT Tests $(0.40 \mathrm{mN} / \mathrm{m})$ being conducted at a pressure of $26.4 \mathrm{MPa}$.

\begin{tabular}{|l|l|l|l|l|l|}
\hline Component & $\underline{\mathrm{C} 1}$ & $\underline{\mathrm{C} 3}$ & $\underline{\mathrm{nC} 5}$ & $\mathrm{nC10}$ & $\underline{\mathrm{nC} 16}$ \\
\hline Mole \% & 82 & 9 & 5 & 2 & 2 \\
\hline
\end{tabular}

Table 2 Composition of 5-component gas 


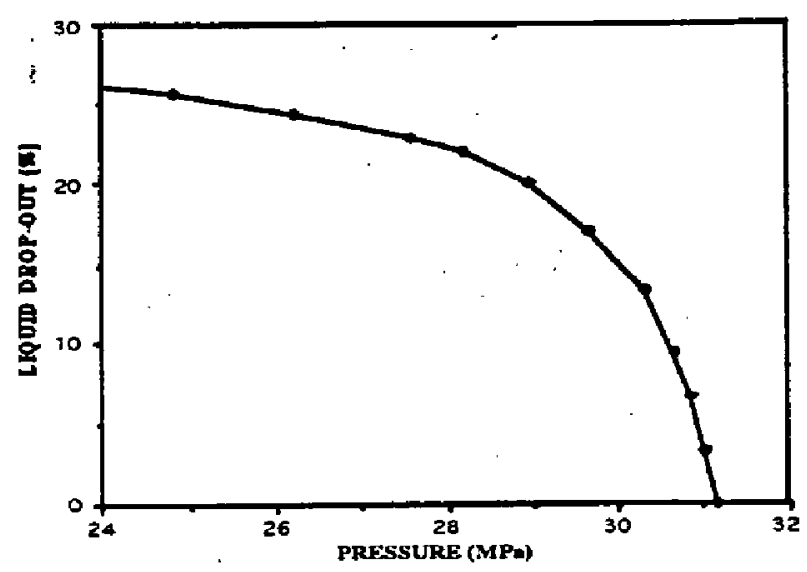

Figure 1 Liquid volume / Dew Point Volume \%, of 5-component gas condensate fluid

\section{Conventional Test fluid}

All conventional unsteady-state displacement tests were conducted using equilibrium methane and butane, gas and oil phases. The phases were equilibrated at the test pressure by flowing them simultaneously through a small bore line.

\section{Gas Condensate Test Procedure}

When below the dew-point in the wellbore region of gas condensate reservoirs, initially the condensate saturation will be established by condensation, with the condensate being immobile due to the pressure reduction and the associated increase in IFT. With distance from the wellbore, the condensate saturation will also be established by the process of condensation. However, as the pressure in the main body of the reservoir is higher, the IFT would be lower in these regions for longer periods. This would allow film flow of condensate to take place towards the wellbore, due to the distribution and flow of condensate being governed mainly by gravitational and capillary forces $[5,7,9]$. The mobile condensate would therefore increase the condensate saturation in the vicinity of the producing wells.

In order to physically simulate these reservoir processes, the follow'ing experimental procedure was adopted. The core was saturated with the single phase gas condensate mixture well above its dew-point. The pressure was subsequently reduced to $30.4 \mathrm{MPa}$, which was $0.83 \mathrm{MPa}$ below the dew-point, allowing an initial condensate saturation of $12 \%$ to form by condensation. No condensate production from the core occurred at this stage, due to the condensate saturation in the core being below the saturation required to initiate flow. In order to increase the condensate saturation in the core and to simulate the reservoir processes, equilibrium gas and condensate were then injected. This resulted in the following procedure being used for each test:

1. The condensate saturation in the core required to initiate the steady-state flow of gas and condensate was established by the simultaneous injection of equilibrium gas and condensate until steady-state conditions were established. During the injection of fluids there was minimal production of condensate from the core outlet, therefore no relative permeabilities were calculated during imbibition, apart from the end-point steadystate values.

2. Equilibrium gas was then injected at the same rate as steady-state flow was established in order to measure recovery of condensate and to establish the residual condensate saturation. The production data was used to calculate the drainage relative permeability.

A total of six tests were conducted following the above procedure, with the relative permeability being measured at three velocities and at two values of IFT. A summary of the test conditions is given in Table 3 .

\section{Conventional Primary Drainage Test Procedure}

Conventional unsteady-state drainage relative permeability curves were generated by saturating the core with equilibrium oil and injecting equilibrium gas. After gas breakthrough the oil and gas relative permeability could be subsequently generated until oil recovery ceased. The objective was to measure the unsteady-state relative permeability by primary drainage with the gas displacement velocity being increased between tests. The test conditions are also shown in Table 3.

\section{Flow rates}

Three flow rates were used in the tests, with the corresponding pore velocities $\left(v_{S}\right)$ calculated as follows;

$$
v_{s}=\frac{q}{A[\phi(1-S w i)]}
$$

Where $q$ is the flow rate, $A$ is the cross sectional area of the core, and $\phi$ is the porosity.

A summary of the flow rates and corresponding capillary numbers $(\mathrm{Nc})$ for all tests is given in Table 3, where $\mathrm{Nc}$ is calculated as;

$$
\dot{N} c=\frac{\mu_{\mathrm{g}} v_{s}}{\sigma}
$$

The calculated Reynolds number ( $R e$ ) ranged between $0.13 \mathrm{E}-4$ to $0.16 \mathrm{E}-2$ indicating that flow was within the laminar flow regime, where $R e$ is calculated as;

$$
\operatorname{Re}=\frac{\rho_{g} \nu_{s} \sqrt{k}}{\mu_{g}}
$$




\begin{tabular}{|c|c|c|c|c|c|}
\hline Tesi & Test Method & $\begin{array}{c}(\mathrm{mN} / \mathrm{m}) \\
\text { IFI }\end{array}$ & $\begin{array}{c}\text { (HCPV/hr) } \\
\text { Flow Rate }\end{array}$ & $\begin{array}{c}\text { (m/day) } \\
\text { Velocity }\end{array}$ & $\begin{array}{c}\text { Capitlary } \\
\text { Number }\end{array}$ \\
\hline 1 & Condensing & 0.05 & 0.06 & 0.88 & $0.94 \mathrm{E}-5$ \\
\hline 2 & Condensing & 0.05 & 0.6 & 8.8 & $0.94 \mathrm{E}-4$ \\
\hline 3 & Condensing & 0.05 & 2.4 & 35.2 & $0.38 \mathrm{E}-3$ \\
\hline 4 & Condensing & 0.40 & 0.06 & 0.88 & $0.90 \mathrm{E}-6$ \\
\hline 5 & Condensing & 0.40 & 0.6 & 8.8 & $0.90 \mathrm{E}-5$ \\
\hline 6 & Condensing & 0.40 & 2.4 & 35.2 & $0.36 \mathrm{E}-4$ \\
\hline 7 & Conventional & 0.14 & 0.6 & 8.8 & $0.28 \mathrm{E}-4$ \\
\hline 8 & Conventional & 0.14 & 2.4 & 35.2 & $0.11 \mathrm{E}-3$ \\
\hline 9 & Conventional & 0.14 & 4.8 & 70.4 & $0.22 \mathrm{E}-3$ \\
\hline
\end{tabular}

Table 3 Summary of test conditions

\section{Relative permeability calculation method}

The relative permeabilities were calculated using the analytical method reported by Bardon \& Longeron [1], based on the fractional flow theory for immiscible fluids during unsteady-state displacements. Although the method has been developed for conventional displacement methods, it is equally valid for condensing systems where only the initial boundary condition is different $[10]$.

\section{RESULTS}

\section{The effect of velocity on relative permeability}

Figures $2 \& 3$ are plots of the gas and condensate relative permeabilities measured during the course of the tests conducted at 0.05 and $0.40 \mathrm{mN} / \mathrm{m}$. The number of data points shown on each curve has been reduced to enhance clarity. The relative permeability curves for the highest rate shown in Figure 3 are based on the extrapolation of the available data points. As the gas and condensate relative permeabilities were measured during the injection of equilibrium gas, they are drainage relative permeabilities. The curves show that the measured relative permeability is sensitive to flow rate, with the relative permeability for both gas and condensate increasing as the velocity increases. The change in the initial steady-state saturation did not vary greatly with changing rate.

At the low IFT conditions of $0.05 \mathrm{mN} / \mathrm{m}$ shown in Figure 2, the rate effect is greater and can be clearly seen for both phases. The increase in gas relative permeability between the lowest and highest rate is approximately three fold at the initial steady-state condition's The associated increase in the condensate relative permeability is greater at approximately four fold. When the IFT was increased to $0.40 \mathrm{mN} / \mathrm{m}$, the relative permeability of both phases reduced as shown in Figure 3, however; the relative permeability rate effect was still evident.

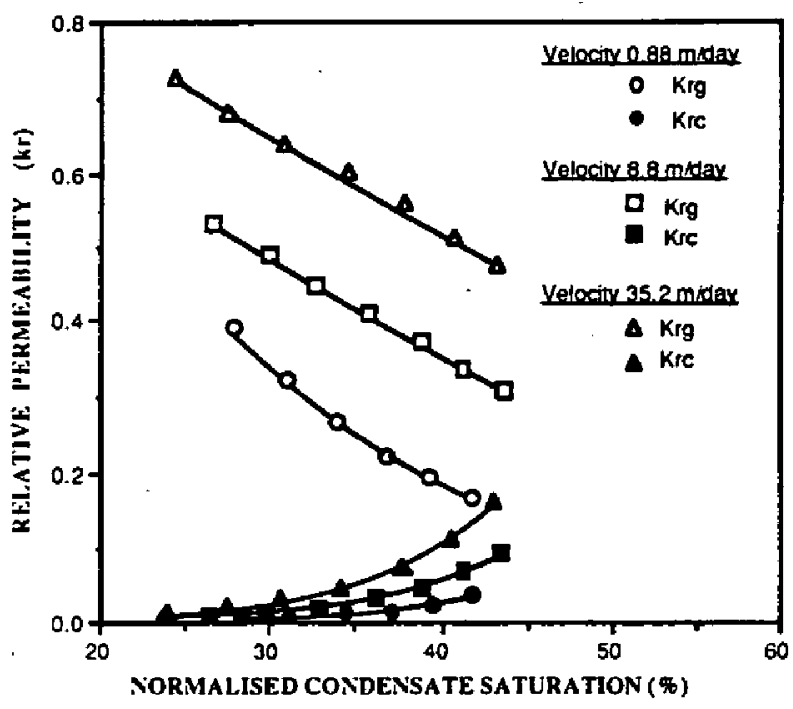

Figure 2 Gas Condensate Relative Permeability (IFT $=0.05 \mathrm{mN} / \mathrm{m}$ )

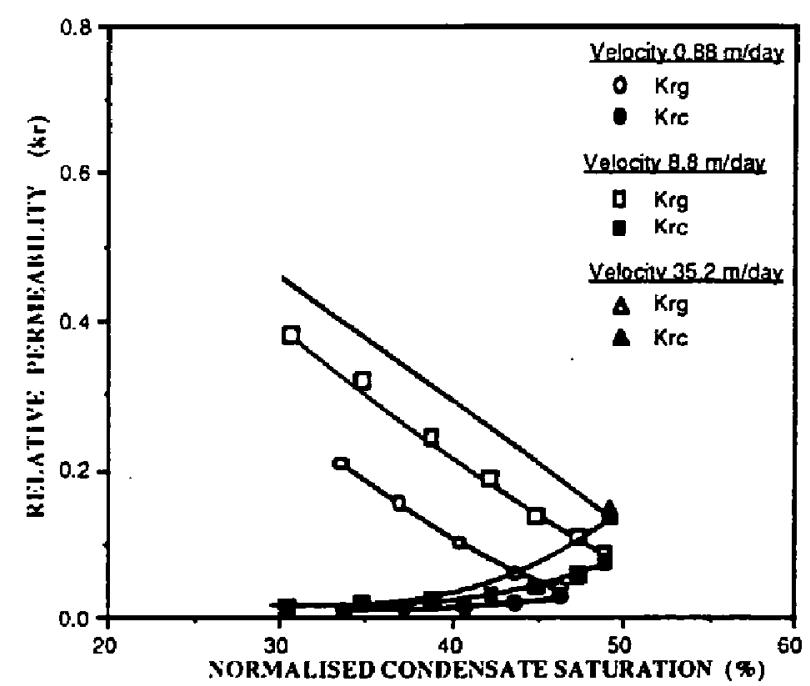

Figure 3 Gas Condensate Relative Permeability (IFT $=0.40 \mathrm{mN} / \mathrm{m}$ ) 


\section{The effect of IFT on relative permeability}

The reduction in gas relative permeability associated with increasing IFT from 0.05 to 0.40 $\mathrm{mN} / \mathrm{m}$ was approximately $75 \%$ at the initial steady-state conditions, for each of the three velocities used in the tests. The corresponding reduction in condensate relative permeability associated with increasing IFT was significantly less than the gas, with the average reduction being only $20 \%$. The initial steady-state condensate saturations increased by an average of $5 \%$ of the HCPV at higher IFT.

The differential pressure across the core reached a maximum of $30 \mathrm{kPa}$ (at a velocity of $35.2 \mathrm{~m} / \mathrm{day}$ ) at an IFT of $0.05 \mathrm{mN} / \mathrm{m}$, and a maximum of $90 \mathrm{kPa}$ (at a velocity of $35.2 \mathrm{~m} /$ day) at an IFT of 0.40 $\mathrm{mN} / \mathrm{m}$. The effect of such pressure differentials on the phase behaviour in the core is believed to be minimal.

In the tests reported, at least eight hydrocarbon pore volumes of gas were injected before the tests were halted. However; in no test was the residual condensate saturation established or even approached, with the condensate saturation reducing by approximately $1 \%$ for every HCPV of gas injected when the tests were terminated. This can be seen in Figure 4 , where condensate production versus 1/HCPV of gas injected is shown for a gas velocity of $35.2 \mathrm{~m} /$ day, at an IFT of $0.05 \mathrm{mN} / \mathrm{m}$. In order to establish the residual condensate saturation during primary drainage at such low IFT values it would require the injection of numerous pore volumes of gas, out with the time scale of these studies.

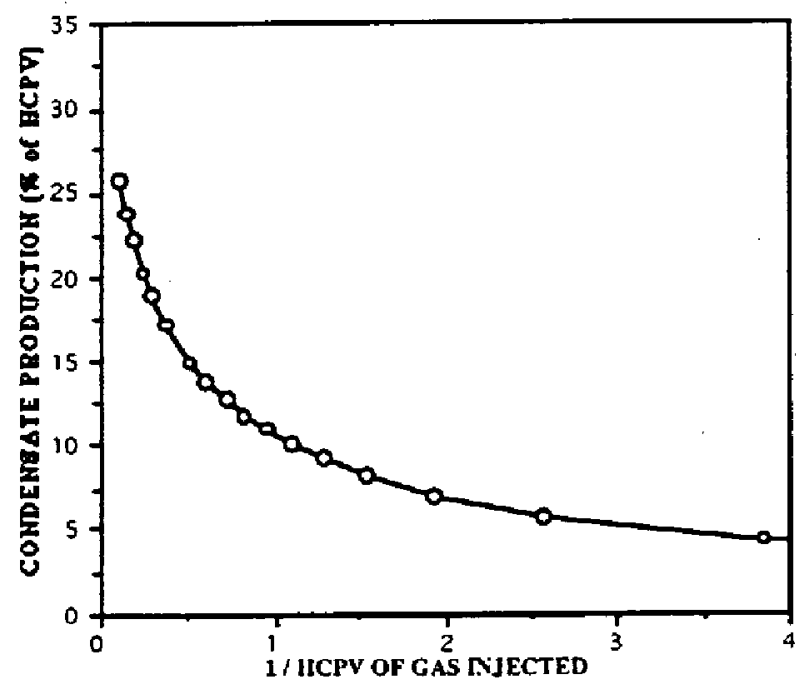

Figure 4 Condensate production versus HCPV of gas injected

The highest flow rate of $35.2 \mathrm{~m} /$ day used in the tests corresponds to a distance of 3 to 5 metres from the wellbore of a typical North Sea gas condensate reservoir.

\section{Conventional gas-oil tests}

Conventional gas-oil displacement tests using the same fluids were conducted to investigate the relative permeability rate effect in the same core at an intermediate IFT of $0.14 \mathrm{mN} / \mathrm{m}$. Three tests were conducted where the core was initially filled with equilibrium oil prior to unsteady-state drainage relative permeability tests being conducted at three different flow rates. The resultant relative permeability curves can be seen in Figure 5 . The sensitivity of relative permeability to velocity is small, and not very significant within the tested range.

As was the case with the condensing fluids, in no test was the residual oil saturation established or even approached, as the oil saturation was reducing by approximately $1 \%$ for every HCPV of gas injected when injection was halted. In contrast, tests reported by Bardon ${ }^{(1)}$ at comparable IFT values have reported that the residual oil saturation was established after the injection of 2 to 4 pore volumes. The velocities used in those tests averaged $4 \mathrm{~m} / \mathrm{day}$, lower than those used in this study.

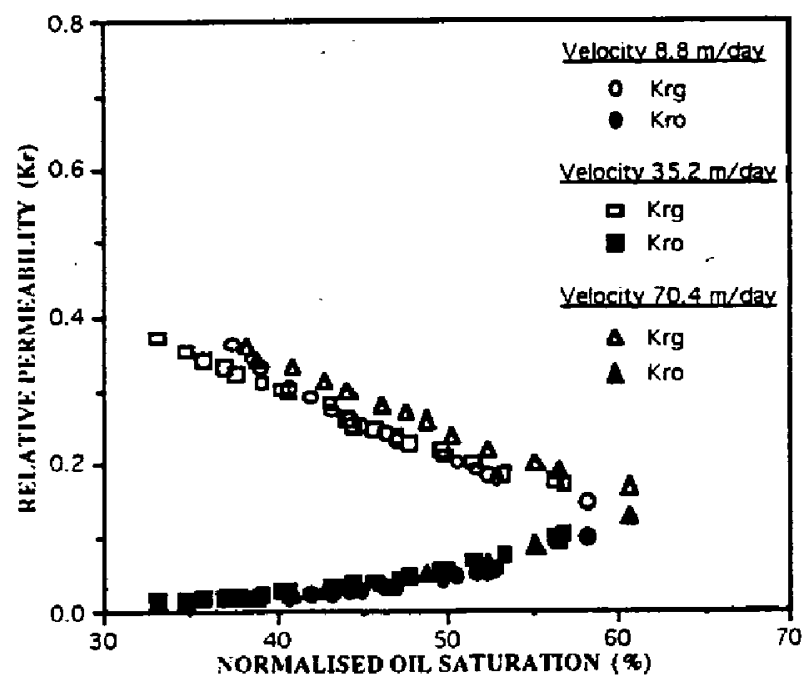

Figure 5 Gas-Oil Relative Permeability (IFT $=0.14 \mathrm{mN} / \mathrm{m}$ )

\section{DISCUSSION}

The influence of core end effects on gas condensate relative permeability

The most significant observation from the tests conducted using condensing fluids was the effect of flow rate on the relative permeability.

Variation in water-oil relative permeability with flow rate has been attributed to core end effects in cores in a publication by Odeh and Dotson [11], where it was reported that during core tests conducted using the unsteady-state dynamic 
displacement technique, that the calculated relative permeability values were strongly influenced by the displacement rates. The influence of end effects on relative permeability was greater at lower flow rates, as the end effect zone in the core increased with decreasing flow rate. This increased the differential pressure caused by the core end effects compared to that caused by viscous forces at low flow rates, resulting in the relative permeability at lower flow rates being underestimated.

A method for estimating the capillary pressure end effect in the non-wetting phase across the outlet face of sandstone cores has been reported by Hassler et al [12], using the following formula;

$$
P c=2.53 \frac{\left(\frac{1-s}{s}\right)}{\sqrt{k}}\left(0.52 \frac{1-s}{s}-0.25\right)
$$

Where $P c$ is the capillary pressure, $s$ is the saturation of the wetting phase (connate water + oil), and $k$ is the permeability.

This is an approximate formula, as the capillary pressure effects can vary between rocks, however; the capillary pressure end effect was calculated for the Berea core used in our study. At the initial steady-state conditions prior to the drainage relative permeabilities being measured, the capillary pressure was calculated to be $0.041 \mathrm{kPa}$ at an IFT of $0.05 \mathrm{mN} / \mathrm{m}$, and $0.31 \mathrm{kPa}$ at 0.4 $\mathrm{mN} / \mathrm{m}$.

In addition to the methods suggested by literature, gas and liquid (condensate) capillary pressure curves were calculated from mercury injection data for the Berea core. For the range of condensate saturations present in the core covered by the relative permeability curves shown in Figures 2 and 3 , the capillary pressure would range from approximately $0.014 \mathrm{kPa}$ at the initial steady-state conditions to $0.07 \mathrm{kPa}$ as the residual condensate saturation was approached at an IFT of 0.05 $\mathrm{mN} / \mathrm{m}$, with the corresponding values ranging from $0.11 \mathrm{kPa}$ to $0.7 \mathrm{kPa}$ at an IFT of $0.4 \mathrm{mN} / \mathrm{m}$.

The measured differential pressure across the core during the tests was two to three orders of magnitude greater than the calculated capilary pressure. The rate effect on relative permeability could therefore not be attributed to end-effects, and would therefore have to be attributable to redistribution of the fluids in the core as the flow rate increased.

\section{Factors governing the gas condensate relative permeability rate effect}

The results have shown that for gas condensate fluids the rate effect is most pronounced at low IFT, and reduces in conjunction with the overall relative permeability as IFT increases. This relationship between flow rate and gas condensate relative permeability in the absence of core end effects has to our knowledge never before been reported, which makes it difficult to compare our findings to previously published gas condensate studies.

Studies conducted by Bardon and Longeron [I] investigated the effect of velocity during unsteadystate relative permeability tests conducted at various values of IFT in long cores using a methane-heptane system. A series of tests were conducted at a fixed IFT of $11 \mathrm{mN} / \mathrm{m}$ where the rate of gas injection varied between tests, with it being concluded that flow rate had no effect on recovery or relative permeability. The absence of any relative permeability sensitivity to velocity may have been attributable to the high IFT prevailing in the tests conducted by Bardon, which was far greater than the IFT between gas and condensate when the relative permeability rate effect was observed. However, the conventional drainage relative permeability tests reported in Figure 5, conducted on the same Berea core as the gas condensate tests, clearly show that no relative permeability rate effect was observed when the IFT was $0.14 \mathrm{mN} / \mathrm{m}$, two orders of magnitude less than the IFT used by Bardon. Clearly therefore it cannot be IFT alone which determines why the rate effect is only observed when using condensing fluids.

Ham and Eilerts ${ }^{[13]}$ have reported that a mobilitysaturation relationship observed in core tests they conducted may have been slightly dependent on velocity. However this was attributed to non-darcy flow. The tests procedures they employed are also not as rigorous as those used in this study.

Our findings suggest that it is the differences in experimental procedure between condensing fluids, the process of establishing the condensate saturation in the core by condensation, and conventional gas-oil fluids, where the fluid saturations are established by injection, that gives rise to the relative permeability rate effect observed for condensing fluids. As no reference could be found in the literature where the condensate saturation in a core was established by condensation, before measuring the relative permeability as a function of flow rate, it is assumed that this is why this relation has never been previously reported.

In general there are two major concepts describing the simultaneous flow of immiscible fluids through pore space. Channel flow occurs when both wetting and non-wetting phases flow through their own separate pore network. Funicular flow describes the simultaneous flow of two fluids in all pores, with the wetting fluid covering and flowing over the grain surfaces, and the non-wetting fluid flowing through the centre of the pores. The situation can be further complicated when three phases are present, as was the case in this study, 
where the intermediate phase (oil/condensate) can spread over the wetting phase (water) and separate the gas and immobile connate water.

During conventional drainage displacements using gas-oil fluids, as reported in Figure 5 of this study, the distribution of oil and the gas is established by the non-wetting phase (gas) displacing oil. Initially the gas will displace oil from the largest available pore space with oil films remaining on the pore walls surrounding the gas channels. The contribution of the oil films to recovery will be minimal as long as networks of oil are continuous in the smaller pore space [14]. Consequently throughout the displacement, oil recovery occurs mainly from pores that contain only oil, with gas flowing through pore space where oil is considered practically immobile.

The channel flow concept used to describe conventional Darcy type flow and fluid distribution in conventional systems is not applicable to condensing fluids, as it has been shown that when below the dew-point the process of condensation will result in condensate forming in all pore space, with condensate flow taking place through a wetting film covering all surfaces of the porous medium [4]. During the displacement of condensate fluids, condensate was observed to flow with gas in all pore space, with both phases flowing together as if they were a single phase $[5$. 15].

The fluid distributions observed for gas condensate fluids resembles the funicular flow concept where the immiscible fluids flow together in all pores. However; it has been reported that for funicular flow, the fluid distributions are independent of velocity [13]. For the tests reported using condensing fluids in our study there was a minimal change in the saturations of both gas and condensate between the three flow rates used at each value of IFT, as shown in Figures $2 \& 3$. Therefore a major difference in fluid distribution between rates is required to explain the reported relationship between relative permeability and velocity. It is believed that it is the process of condensation in conjunction with the flow characteristics of condensing fluids which accounts for the different relative permeability behaviour reported in this paper. A new flow concept may therefore be required to adequately explain the flow of condensing fluids and the associated relative permeability sensitivity to velocity.

The sensitivity of relative permeability to velocity for condensing fluids is believed to be a new and significant finding. The results reported raise doubts over the transfer. of conventional gas-oil data to flow of gas condensate fluids, as this data would under predict the mobility of gas condensate fluids at high displacement velocities, especially at low IFT. This study has highlighted the need for experimental procedures to be employed when using condensing fluids which will be representative of fluid distributions in gas condensate reservoirs.

Although the data reported can be used when planning future gas condensate reservoir management, this area still requires further investigation which is already underway. The reduction in the relative permeability rate effect as IFT increases indicates that at higher values of IFT the rate effect could disappear, and is clearly worth further investigation.

\section{CONCLUSIONS}

I. Tests conducted using gas condensate fluids and appropriate experimental procedures have shown that relative permeability was rate sensitive. The relative permeabilities of both phases were found to increase as the velocity increased.

2. As IFT increased, the gas and condensate relative permeabilities reduced, however the rate effect was still evident, with the gas relative permeability reducing more than the condensate.

3. The rate effect was shown to be independent of core end effects.

4. The relative permeability rate effect is attributed to the process of condensation in conjunction with the flow characteristics of gas condensate fluids. This leads to redistribution of fluids as the flow rate increases with minimal change in saturation.

5. Relative permeability tests conducted at similar conditions on the same core using conventional experimental procedures exhibited a negligible rate sensitivity. Data generated using conventional relative permeability procedures is therefore not directly transferable to gas condensate systems.

6. In order to accurately measure the relative permeability of gas condensate fluids, experimental procedures are required which will be representative of the fluid distributions in gas condensate reservoirs.

\section{ACKNOWLEDGEMENTS}

The authors wish to gratefully acknowledge the financial support for this research programme provided by; British Gas plc, British Petroleum Exploration Operating Co ple, Chevron UK Ltd, Conoco UK Ltd, DTI, Elf Exploration UK Ltd, Marathon Oil UK Ltd, Mobil North Sea Ltd, Phillips Petroleum Company UK Ltd, and Shell UK Exploration and Production, Total Oil Marine plc. 


\section{NOMENCLATURE}

A - Area.

$\rho_{\mathrm{g}}$ - Gas density.

$\phi$ - Porosity.

IFT - Interfacial tension.

k - Permeability.

$\mu_{\mathrm{g}}$ - Gas viscosity.

$\mathrm{N}_{\mathrm{C}}$ - Capillary number.

q - Flow rate.

Re - Reynolds number.

$\sigma$ - Interfacial tension.

Swi - Connate water saturation.

$u_{S}$ - Pore velocity.

\section{REFERENCES}

1. Bardon, C. and Longeron, D.G. : "Influence of Very Low Interfacial Tension on Relative Permeability", SPE J., Oct, 1980.

2. Assar, J. and Handy, L.L. : "Influence of Interfacial Tension on Gas/Oil Relative Permeability in a Gas Condensate System", SPE, Reservoir Engineering, 1988.

3. Haniff. M.S., \& Ali J.K. “ Relative Permeability and Low Tension Fluid Flow in Gas Condensate Systems" SPE 20917, Europec conf. The Hauge, 1990.

4. Danesh, A., Henderson, G.D., Peden, J.M. : "Experimental Investigation of Critical Condensate Saturation and its Dependence on Connate Water Saturation in Water Wet Rocks", SPE 19695, Ann. Tech. Conf., San Antonio, 1989.

5. Henderson, G.D.., Danesh.A., Tehrani, D.H., Peden.J.M., :" An Investigation into the Processes Governing Flow and Recovery in Different Flow Regimes Present in Gas Condensate Reservoirs", SPE, Ann. Tech. Conf. Houston, 1993.

6. Henderson, G.D., Danesh.A., Tehrani, D.H., Peden.J.M., :" An Experimental

Investigation of Waterflooding of Gas Condensate Reservoirs and their Subsequent Blowdown" J. of Pet. Science and Eng. 8, 1992.

7. Danesh.A., Henderson.G.D., Krinis.D.,

Peden.J.M., :" Experimental Investigation of Retrograde Condensation in Porous Media at Reservoir Conditions" SPE 18316, 63rd Ann.Tech.Conf., Houston 1988.

8. Danesh, A., Todd, A.C., Somerville, J. \& Dandekar, A. "Direct Measurement of Interfacial Tension, Density, Volume and Compositions of Gas Condensate Systems" Trans. IChemE, Vol 68, Part A, 1990.
9. Henderson G.D. "Gas Condensate Recovery by Gravity Drainage and Flooding Processes" Thesis, Dept, of Pet. Eng., Heriot-Watt Univ. 1994.

10 M. Khazam. "Application of Three Phase Behaviour and Flow Models to Gas Injection and Gas Condensate Processes" Thesis, Dept, of Pet. Eng., Heriot-Watt Univ. 1994.

11. Odeh, A.S. and Dotson, B.J. :"A Method For Reducing The Rate Effect On Oil and Water Relative Permeabilities From Dynamic Displacement Data" Journal of Petroleum Tech, ,Nov. 1985.

12. Hassler, G.L., Brunner, E., Deahl, T.J., :"The Role of Capilliarity in Oil Production", T.P. 1623, Petroleum Technology 1943.

13. Ham, J.D. \& Eilerts, C.K. "Effect of Saturation on Mobility of Low LiquidVapour Ratio Fluids" Trans AIME p11-19, 1967.

14. Dullien. F.A.L. "Porous Media : Fluid Transport and Pore Structure" Academic Press, 2nd Edition, 1992.

15. Henderson.G.D., Danesh.A., Tehrani, D.H., Peden.J.M., :" Waterflooding of Gas Condensate Fluids in Cores Above and below the Dew-Point. " SPE, 66th, Ann. Tech. Conf, Dallas, Oct. 1991. 